\title{
Role of the fixed combination of fluticasone and salmeterol in adult Chinese patients with asthma and COPD
}

REVIEW

This article was published in the following Dove Press journal:

International Journal of COPD

15 April 2015

Number of times this article has been viewed

Jinming Gao'

Roy A Pleasants ${ }^{2}$

'Department of Respiratory Diseases, Peking Union Medical College Hospital, Peking Union Medical College and Chinese

Academy of Medical Sciences, Beijing, People's Republic of China; ${ }^{2}$ Division of Pulmonary, Allergy, and Critical Care Medicine, Department of

Medicine, Duke University Medical

Center, Durham, NC, USA
Correspondence: Jinming Gao Department of Respiratory Diseases, Peking Union Medical College Hospital, I Shuaifuyuan, Dongcheng District, Beijing 100730, People's Republic of China

$\mathrm{Tel}+861069155049$

Fax +86 I065I2 4875

Email gjinming@yahoo.com
Abstract: Chronic obstructive pulmonary disease (COPD) and asthma are common airway disorders characterized by chronic airway inflammation and airflow obstruction, and are a leading cause of morbidity and mortality in the People's Republic of China. These two diseases pose a high economic burden on the family and the whole of society. Despite evidence-based Global Initiative for Chronic Obstructive Lung Disease and Global Initiative for Asthma guidelines being available for the diagnosis and management of COPD and asthma, many of these patients are not properly diagnosed or managed in the People's Republic of China. The value of combination therapy with inhaled corticosteroids and long-acting $\beta_{2}$-agonists has been established in the management of asthma and COPD globally. Combinations of inhaled corticosteroids and long-acting $\beta_{2}$-agonists such as fluticasone and salmeterol, have been shown to be effective for improving symptoms, health status, and reducing exacerbations in both diseases. In this review, we discuss the efficacy and safety of this combination therapy from key studies, particularly in the People's Republic of China.

Keywords: People's Republic of China, chronic obstructive pulmonary disease, asthma, fluticasone, salmeterol

\section{Background}

Chronic obstructive pulmonary disease (COPD) and asthma are common airway disorders characterized by chronic airway inflammation and airflow obstruction that often require pharmacological agents with complementary mechanisms of action. ${ }^{1-3}$ For more than 15 years, the value of inhaled corticosteroids (ICS) and long-acting $\beta_{2}$-agonist (LABA) combination products has been established in the management of asthma and COPD, ${ }^{4-15}$ and has had a substantial impact on the management of these diseases globally. The Global Initiative for Chronic Obstructive Lung Disease (GOLD) and Global Initiative for Asthma (GINA) guidelines provide evidence-based recommendations for the use of ICS/LABA in COPD, asthma, and more recently for asthma/COPD overlap syndrome. .,2,16 $^{2}$

The aim of this review is to summarize the combination therapy of the ICS fluticasone propionate (FP) and the LABA salmeterol (SAL) for the treatment of COPD and asthma, specifically as relevant to the adult Chinese population. In addition to retrieving studies concerning the epidemiology of COPD and asthma in the People's Republic of China, we performed a PubMed search using the key terms "fluticasone", "salmeterol", "asthma", and "COPD". We present the relevant pivotal and key clinical trials evaluating the efficacy and safety of FP/SAL, as well as comparative studies with other prescription medications that are widely used in the People's Republic of China for obstructive lung diseases. 


\section{Epidemiology of COPD and asthma in the People's Republic of China Prevalence}

In the People's Republic of China, COPD is increasingly becoming a major public health problem and is one of the leading causes of disability. ${ }^{17,18}$ According to the large epidemiological studies, the overall prevalence of COPD in adults in the People's Republic of China was reported to be $8.2 \%{ }^{19}$ and the hospitalization rate approximately $1.6 \%{ }^{20} \mathrm{COPD}$ was found to be the fourth leading cause of mortality in rural areas of the People's Republic of China, with a prevalence of $4.4 \%-16.7 \%$. In urban areas of the People's Republic of China, it was the third leading cause of mortality, with a prevalence rate of $6.7 \%-8.3 \% .{ }^{21}$ It is projected that more than 65 million Chinese people will die prematurely of COPD between 2003 and 2033. ${ }^{22}$ Major risk factors for developing COPD in the People's Republic of China are tobacco smoking, use of biomass fuels for heating and cooking, and air pollution. ${ }^{22}$ In the People's Republic of China, men are 2-3 times more likely to have COPD than women because of relatively higher smoking rates in men. ${ }^{23}$ However, more biomass fuel exposure is seen in women..$^{21,24}$ A 2010 report on tobacco use rates in the People's Republic of China indicated that $28.1 \%$ of all adults were current smokers ( $52.9 \%$ of men and $2.4 \%$ of women); the highest rates were in men aged $45-64$ years $(63.0 \%) .{ }^{24}$ Similar to studies in other countries, persons with low educational and household income levels are more prone to developing COPD, whether or not associated with tobacco use. ${ }^{25}$ Apart from these, malnutrition, respiratory infections, and physical inactivity contribute to an increased risk of COPD. ${ }^{21}$

Asthma is also a major cause of health resource utilization, poor health-related quality of life, and disability for affected persons. The global prevalence of asthma diagnosis is reported to be $4.3 \%$, ranging from $0.2 \%$ in the People's Republic of China to $21 \%$ in Australia. ${ }^{2}$ It is estimated that nearly 300 million persons worldwide have asthma, and it is projected that by 2025 , there will be nearly 400 million persons with the disease. ${ }^{2}$ Unfortunately, one in five of those with clinical asthma did not receive treatment for their asthma. ${ }^{26}$ In the People's Republic of China, the reported prevalence of asthma is lower than in the West. Within the People's Republic of China, the prevalence varies among regions; in Hong Kong, $12.4 \%$ and $10.1 \%$ of children aged 13 and 14 years had wheeze compared with $4.2 \%$ and $2.0 \%$ in similarly aged children in the People's Republic of China..$^{27,28}$ The lower rates of asthma in the People's Republic of China, compared with countries such as the USA, may be related to lower rates of allergy in the Chinese population. ${ }^{29}$ In addition, other potential contributors to the lower asthma prevalence may include underdiagnosis, genetics, and possibly development of immunity in response to the environment in the People's Republic of China (asthma hygiene hypothesis)..$^{30}$

\section{Economic burden}

There are substantial costs associated with treatment of COPD in the Chinese patient. A survey in the People's Republic of China conducted in 2006 in more than 700 COPD outpatients from different cities found that the mean annual direct medical cost (ie, medicines and hospitalization) was $11,744 \mathrm{RMB}$ yuan $(\sim 1,730)$ and the direct nonmedical cost (ie, absence from work) was 1,570 RMB yuan ( $\$ 230)$ in the urban patient. ${ }^{31}$ The total expenditure for each COPD patient accounted for $40 \%$ of an average family's income. Another survey in 7,383 COPD patients in rural areas of the People's Republic of China estimated the direct costs to be 1,090 RMB yuan $(-\$ 175)$ per person. This was primarily the result of costs for outpatient services (42.3\%), hospitalizations $(32.5 \%)$, and medicines $(20.5 \%)$. The indirect costs per COPD patient were estimated to be more than 20,000 RMB yuan (nearly $\$ 3,200$ ). In rural areas of the People's Republic of China, the health care costs of COPD accounted for nearly one third of a typical family's income. ${ }^{32}$ This financial burden likely contributes to suboptimal treatment of COPD in the People's Republic of China, particularly in low-income persons.

\section{Current status of COPD and asthma in the People's Republic of China}

Published international and Chinese strategies for COPD and asthma encourage physicians to adopt the GOLD and GINA recommendations for the management of these two prevalent airway diseases. ${ }^{1,2,18,33}$ As with many disease guidelines, their implementation is challenging for a variety of reasons. The diagnosis of COPD is often delayed in the People's Republic of China because of physicians not identifying risk factors, addressing symptoms, and/or assessing biomarkers. ${ }^{34,35}$ Only one in five primary care physicians are appropriately knowledgeable about the GOLD recommendations for pharmacological management in COPD. ${ }^{36}$ In the People's Republic of China, about two thirds of COPD patients show at least one respiratory symptom (cough, phlegm, wheezing, and dyspnea), whereas about one third of COPD patients had airflow obstruction by spirometry but were asymptomatic. ${ }^{19}$ Most physicians in the People's Republic of China diagnose COPD without performing spirometry to confirm the disease or determine its severity, with less than one-third 
performing spirometry on one study. ${ }^{37}$ The medications prescribed by physicians for the Chinese COPD patient often differ from the GOLD strategy recommendations, as most of the frequently prescribed medications are expectorants, short-acting inhaled bronchodilators $\beta_{2}$-agonists and anticholinergics. ${ }^{37}$

Although there have been improvements in overall asthma control as defined by GINA in recent years in the People's Republic of China, the current level of asthma control at the national level continues to fall behind the international standard defined by GINA. Su et al conducted a national survey to study the association between baseline information on asthma control and patients' perception of asthma. A face-toface, questionnaire-based survey was conducted in $>3,000$ asthmatic patients from 36 general hospitals located in ten geographically dispersed cities over a one-year period. ${ }^{38} \mathrm{In}$ this survey, only $28.7 \%$ and $45.0 \%$ of the patients achieved control or partial control, respectively, $21.8 \%$ used peak flow meters, and $6.6 \%$ of patients used peak flow meters daily. ICS plus LABA and ICS alone were used in $45.6 \%$ and $30.4 \%$ of patients, respectively. The number of sick days, emergency department visits, and hospitalizations were significant in the Chinese population with asthma. Achieving control in the majority of asthmatics is a global problem not unique to the People's Republic of China.

\section{Effect of FP/SAL on airway and systemic inflammation}

An example of the synergistic molecular effects of SAL and FP in inhibiting airway inflammation has been described. Barnes et al measured the change in infiltration of inflammatory cells into bronchial tissues before and after FP/SAL therapy. ${ }^{39}$ Compared with placebo treatment, $\mathrm{CD} 8{ }^{+} \mathrm{T}$-cells were decreased by $36 \%$ from baseline with FP/SAL $(P=0.02)$ and $\mathrm{CD} 4^{+}$T-cells by $40 \%(P=0.017)$. Neutrophil counts declined slightly in the FP/SAL-treated group.

Evidence supporting an effect of ICS in inhibiting the systemic inflammation found with COPD was shown by a study of serum C-reactive protein (CRP), which is typically elevated in COPD. ${ }^{40}$ In a study where the ICS was withdrawn in a group of COPD patients, serum CRP levels increased, while serum CRP and interleukin- 6 levels decreased by $50 \%$ and $26 \%$ respectively, after 2 weeks of inhaled FP. Sin et al completed the large ABC (Advair Biomarkers in COPD) study, which assessed the effects of FP alone or in combination with SAL on systemic inflammation in patients with COPD.$^{41}$ The data showed that ICS with or without a LABA significantly reduced levels of serum surfactant protein-D, a lung-specific biomarker of systemic inflammation, in patients with COPD.

Yang et al studied the anti-inflammatory effects of FP/SAL $500 / 50 \mu \mathrm{g}$ in 21 Chinese patients with moderate or severe COPD ${ }^{42}$ These authors demonstrated that FP/SAL significantly reduced the levels of inflammatory cytokines, interleukin-17A, interleukin-8, and tumor necrosis factor-alpha, in both sputum and blood. Meanwhile, the proportion of Foxp $3^{+}$regulatory $\mathrm{T}$-cells in the total $\mathrm{CD} 4^{+} \mathrm{T}$-cell population in peripheral blood was significantly increased after treatment with FP/SAL.

A 6-month, open-label clinical trial was conducted in 122 Chinese patients with stable moderate or severe COPD, aiming to compare the difference in anti-inflammatory effects between the combination of FP/SAL 500/50 $\mu \mathrm{g}$ twice daily and the combination of albuterol and ipratropium four times daily. ${ }^{43}$ Compared with short-acting bronchodilators, the combination of FP/SAL significantly reduced CRP levels $(-1.73 \mathrm{mg} / \mathrm{L}$ versus $0.08 \mathrm{mg} / \mathrm{L}$ ) at the end of treatment. Consistently, patients on treatment with FP/SAL in whom CRP decreased had a significantly higher improvement in forced expiratory volume in one second $\left(\mathrm{FEV}_{1}\right)$ and St George Respiratory Questionnaire total scores than those in whom CRP did not decrease. In another study, Du et al reported that 12 weeks of treatment with FP significantly decreased sputum levels of tumor necrosis factor-alpha and neutrophils in Chinese patients with COPD. ${ }^{44}$

ICS are the first-line therapy for asthma management because of their substantial efficacy in inhibiting asthmatic airway inflammation. ${ }^{2}$ In addition, LABA have been shown to have ICS-sparing effects. ${ }^{14,15}$ Several studies have evaluated the effect of the FP/SAL combination on markers of airway inflammation in patients with asthma. ${ }^{45,46} \mathrm{Currie}$ et al measured several noninvasive markers of airway inflammation in 25 patients with moderate asthma. Compared with SAL alone, FP/SAL provided significant improvements in all surrogate markers of airway inflammation $(P<0.05)$, with the exception of serum eosinophil cationic protein..$^{45}$ Another study using induced sputum from 66 asthmatics showed a trend for a decreased neutrophil count with 12 weeks of treatment with FP/SAL. ${ }^{46}$

\section{Guideline-based therapies for asthma and COPD}

The asthma guidelines recommend ICS as first-line maintenance therapy for persistent asthma and for asthmatics who require use of short-acting $\beta_{2}$-agonists more than once daily. ${ }^{2}$ Treatment with an ICS/LABA combination is often reserved for asthma patients inadequately managed with ICS alone. In the People's Republic of China, the two proprietary ICS/ LABA combinations are FP/SAL (Seretide ${ }^{\circledR}$ Accuhaler $^{\circledR}$ 
and metered dose inhaler [MDI]) and budesonide/formoterol (Symbicort Turbuhaler ${ }^{\circledR}$ and MDI). Treatment with a regular daily low dose of ICS is highly effective in reducing asthma symptoms in most patients, along with the risk of asthma-related exacerbations, hospitalizations, and death. ${ }^{2}$ In addition, early initiation of low-dose ICS in patients with asthma provides greater improvement in lung function than if symptoms have been present for more than 2-4 years. ${ }^{2}$ Oral montelukast (Singulair ${ }^{\circledR}$ ) is also recommended as antiinflammatory maintenance medication for the treatment of persistent asthma, particularly in patients with concurrent allergic rhinitis. ${ }^{2}$ Theophylline, a mild bronchodilator and anti-inflammatory agent, is recommended as a second-line or third-line agent for chronic asthma.

The Respiratory Diseases Branch of the Chinese Medical Association also recommends ICS as first-line maintenance therapy for asthma and ICS/LABA to be used in patients with moderate-to-severe disease. ${ }^{33}$ One study in the Chinese population showed overall asthma control was comparably achieved between patients receiving half the dose of ICS recommended by GINA and those receiving the GINA-defined high dose of ICS. ${ }^{47}$ This investigation suggests that a lower dosage of ICS might be preferred in many Chinese patients with asthma.

According to the 2014 GOLD COPD strategy document, bronchodilators are the principal therapy for symptomatic management of COPD patients. ${ }^{1}$ The long-acting agents, ie, LABAs and long-acting muscarinic antagonists, have been shown to be more effective for symptom relief and are also more conveniently dosed than the short-acting bronchodilators. Two twice-daily inhaled LABAs (SAL and formoterol) have been available for the treatment of COPD for over 20 years. More recently, indacaterol, a once-daily LABA, was approved in the People's Republic of China for COPD. SAL provides significant improvement in $\mathrm{FEV}_{1}$, dyspnea, health-related quality of life, need for rescue medication, and exacerbation rates. ${ }^{12,48,49}$ Theophylline is recommended as a third-line or fourth-line agent in COPD, and in addition to its bronchodilating and anti-inflammatory effects, reduced fatigue of accessory breathing muscles may be relevant in COPD. ${ }^{1}$

The GOLD guidelines recommend long-acting bronchodilators as preferred maintenance treatment for moderate COPD and adding ICS as indicated. ${ }^{1}$ The combination of an ICS/LABA and a long-acting muscarinic antagonist, also referred to as triple therapy, is a common regimen used in patients with advanced COPD. According to GOLD, the combination of an ICS with a LABA is reserved for patients in GOLD groups C and D, specifically those at increased risk of exacerbations with severe or very severe airflow obstruction and in patients with COPD-asthma overlap syndrome. ${ }^{1,16}$
The Respiratory Diseases Branch of the Chinese Medical Association guidelines recommend bronchodilators as firstline treatment for COPD patients and ICS/LABA for symptomatic patients with $\mathrm{FEV}_{1}$ being less than $50 \%$ predicted and those with frequent exacerbations. ${ }^{18}$

\section{Clinical efficacy and safety of FP/SAL in COPD and asthma Clinical trials of FP/SAL in COPD}

Although the key clinical trials for FP/SAL were not conducted in the Asian population, they serve as a basis for their efficacy and safety in humans and are therefore included in this review. Table 1 shows the prospective, randomized clinical trials evaluating the efficacy and safety of the FP/SAL combination when compared with its individual ingredients. First, the pivotal trial by Hanania et al which helped to secure US Food and Drug Administration approval, was a placebocontrolled evaluation of the FP/SAL 250/50 $\mu \mathrm{g}$ dry powder inhaler (DPI) twice daily, compared with the individual agents at the same doses in a group of COPD patients, largely with moderate or severe airflow obstruction. ${ }^{13}$ There were also two other placebo-controlled studies published around the same time that included patients with very severe airflow obstruction, but used the higher doses of FP/SAL (500/ $50 \mu \mathrm{g}){ }^{9,}{ }^{10}$ The primary endpoint in all of these studies was lung function $\left(\mathrm{FEV}_{1}\right)$, and in each instance the FP/SAL combination was superior to the individual ingredients. For the secondary endpoints in these studies, typically health status, symptoms, and acute exacerbations of COPD, there was no consistent pattern in outcomes. For two of the three studies, there was no difference between FP/SAL and the individual agents with regard to risk of acute exacerbations of COPD. ${ }^{9,13}$ In the other study, only FP/SAL was superior to placebo. ${ }^{10}$ In the two studies that included dyspnea as a measure, one showed FP/SAL was superior to placebo and the individual agents, ${ }^{9}$ but this was not seen in another study. ${ }^{13}$ In two later studies where acute exacerbations of COPD were the primary study endpoint, FP/SAL was superior to SAL alone. ${ }^{50,51}$

TRISTAN (Trial of Inhaled Steroids and Long-acting $\beta 2$-Agonists) was a key clinical trial comparing the effects of FP and SAL used as monotherapies or in combination. FP/SAL combination therapy improved pretreatment $\mathrm{FEV}_{1}$ significantly more than did FP or SAL alone $(95 \mathrm{~mL}$ and $73 \mathrm{~mL}$, respectively, all $P<0.0001) .{ }^{10}$ The combination therapy produced a clinically significant improvement in health status and the most substantial improvement in daily symptoms. Subanalysis data showed that these effects were more obvious in patients whose baseline $\mathrm{FEV}_{1}$ was $<50 \%$ predicted. 


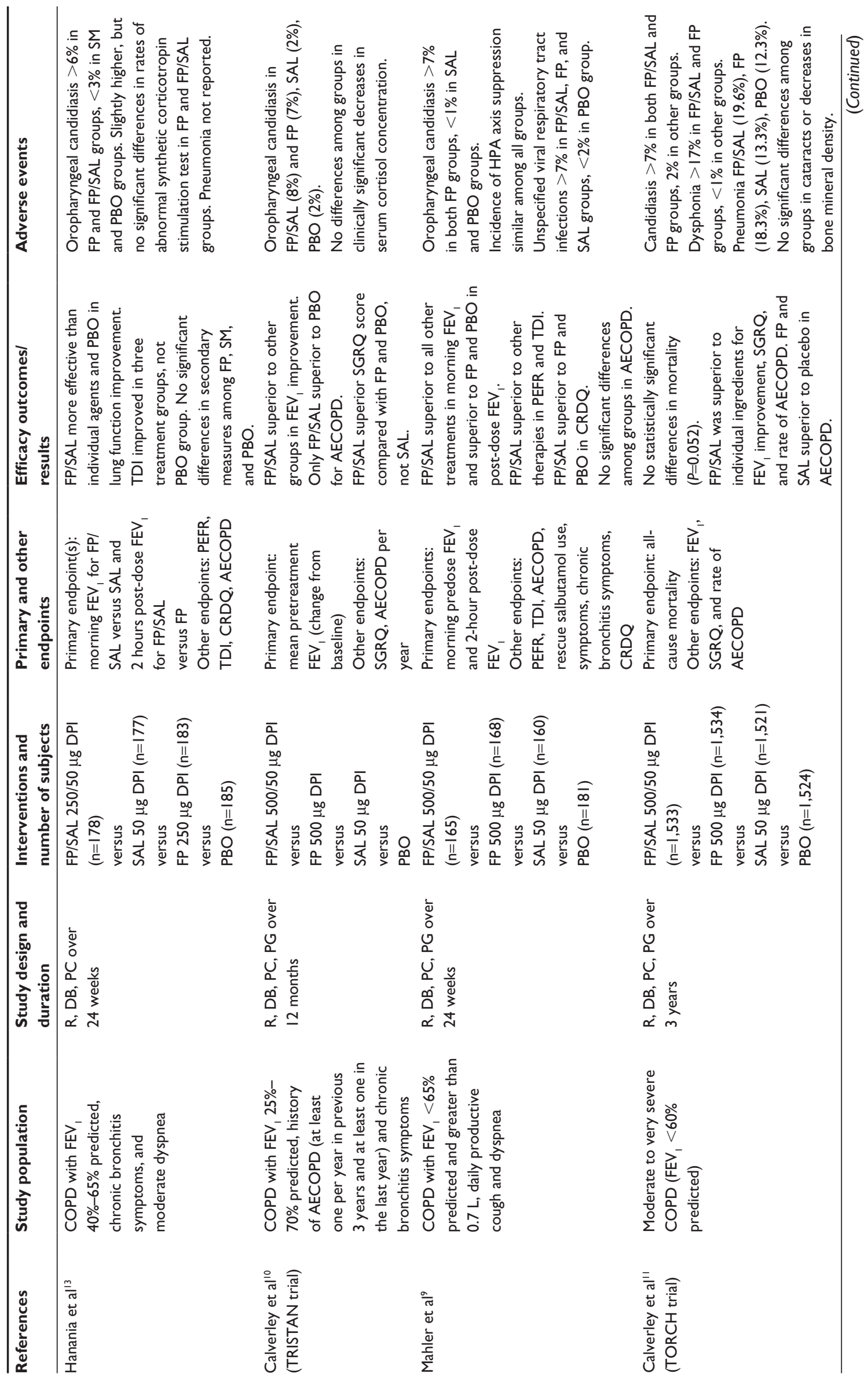




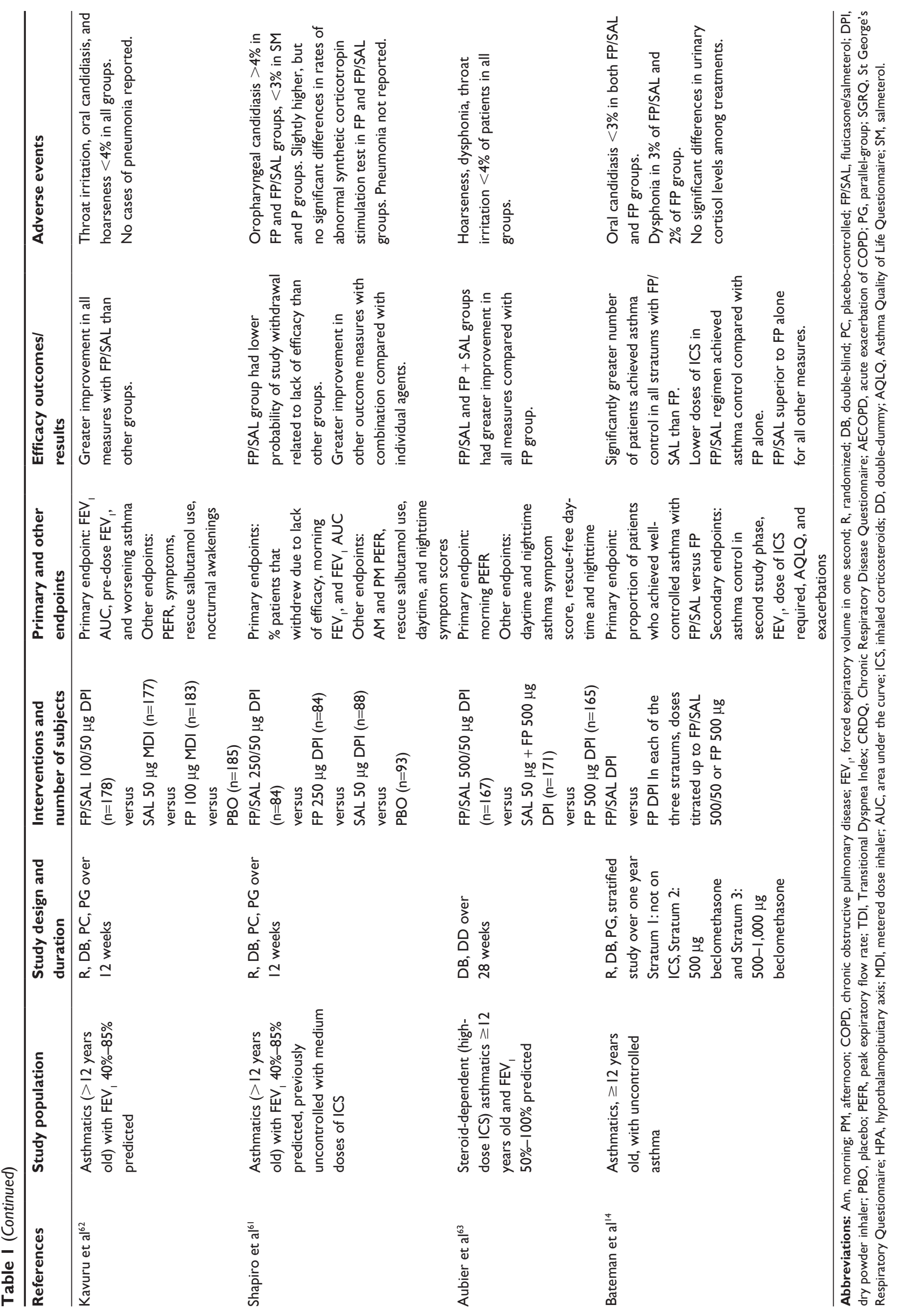


TORCH (Towards a Revolution in COPD Health) was a landmark study designed as a 3-year, double-blind, parallelgroup, placebo-controlled investigation in 6,184 COPD patients randomized to receive twice-daily SAL $50 \mu \mathrm{g}$ and FP $500 \mu \mathrm{g}$, either as monotherapy or in combination. ${ }^{11}$ The primary outcome was all-cause mortality, with additional post hoc analysis including the effect of the therapies on the rate of decline in $\mathrm{FEV}_{1}$. TORCH did not find a statistically significant decrease in mortality among patients treated with FP/SAL combination therapy when compared with placebo (hazard ratio $0.825, P=0.052$ ). The implication of making clinical decisions about the impact of a drug with regard to decreased mortality when the $P$-value is so close to 0.05 is worthy of debate. Active treatments did reduce the rate of exacerbations compared with placebo $(P<0.001)$, and exacerbations requiring hospitalization were decreased with the FP/SAL combination and SAL alone when compared with placebo $(P<0.03)$. The combination therapy also improved health-related quality of life compared with placebo and either therapy alone. Sustained improvement in lung function measures was observed in all active treatment groups compared with placebo.

There have been a number of post hoc analyses done with the TORCH study results. One post hoc analysis evaluated the effects of FP, SAL, and FP/SAL compared with placebo on the rate of decline in $\mathrm{FEV}_{1}{ }^{12}$ For the placebo group, the rate of $\mathrm{FEV}_{1}$ decline was $55 \mathrm{~mL}$ per year, whereas it was $39 \mathrm{~mL}$ per year in the ICS/LABA group and $42 \mathrm{~mL}$ per year in the FP and SAL groups $(P<0.03)$. A later publication also showed that subjects in the TORCH study who were $>80 \%$ adherent with use of FP/SAL had substantially lower hospitalization and mortality rates, thus supporting the need for health care providers to encourage medication adherence with this therapy. ${ }^{52}$ Finally, in another post hoc analysis that evaluated the effects of FP/SAL combination therapy based upon GOLD severity classification, there was a statistically significant decrease in mortality associated with the FP/SAL combination in GOLD stage II (moderate) but not GOLD stage III or IV disease. ${ }^{53}$

Zheng et al studied the efficacy and tolerability of FP/SAL (500/50 $\mu \mathrm{g}$ twice daily) in the treatment of Chinese patients with COPD over 24 weeks (Table 2). ${ }^{54}$ This was a randomized, double-blind, placebo-controlled, parallel-group, multicenter study. Primary endpoints included changes in pre-FEV ${ }_{1}$ and post-FEV ${ }_{1}$. Compared with the placebo group, the FP/SAL group showed higher mean changes in pre-FEV $(180 \mathrm{~mL}, P<0.001)$ and post-FEV ${ }_{1}(65 \mathrm{~mL}, P<0.012)$. The benefit of $\mathrm{FP} / \mathrm{SAL}$ was more apparent in former or current smokers. FP/SAL significantly improved life quality and reduced the frequency of nocturnal awakenings and use of as-needed bronchodilators. Exacerbations of COPD were also decreased in the FP/SAL group relative to the placebo group $(P<0.01)$.

The recommended dose of $\mathrm{FP} / \mathrm{SAL}$ for COPD in the USA is 250/50 $\mu \mathrm{g}$ twice daily (Advair ${ }^{\circledR}$ Diskus $^{\circledR}$ ) and 500/ $50 \mu \mathrm{g}$ twice daily in the UK and the People's Republic of China (Seretide Accuhaler ${ }^{\circledR}$ ). There is one published study directly comparing FP/SAL 500/50 $\mu \mathrm{g}$ and FP/SAL 250/50 $\mu \mathrm{g}$, which was conducted in an Asian population. ${ }^{55}$ This study showed, in a group of Taiwanese patients, that the higher dose provided greater improvement in $\mathrm{FEV}_{1}$ and health status as well as fewer acute exacerbations of COPD. Further, there was no difference in the risk of pneumonia between the treatment groups. Among placebo-controlled studies that did not directly compare the two doses of FP/SAL, there was little difference in the effect on symptoms between the $500 \mu \mathrm{g}^{13}$ and $1,000 \mu \mathrm{g}$ per day doses, ${ }^{9-11,54}$ suggesting a flat dose-response for FP for this measure. An appropriate dosage of FP in combination with SAL for COPD management needs to be clarified, considering the benefits and potential short-term and long-term side effects of ICS. ${ }^{7}$

\section{Comparative studies of FP/SAL and tiotropium in COPD}

A number of studies have evaluated the efficacy and safety of FP/SAL compared with tiotropium (TIO), either alone or in combination, in COPD (Tables 3 and 4). ${ }^{56-60} \mathrm{TIO}$ is not approved for asthma in the People's Republic of China, so we did not include published studies that have evaluated the role of this agent in asthmatics, such as in combination with ICS and/or LABA. As shown in Tables 2 and 3, the primary outcomes in these studies were lung function measures ${ }^{56,58}$ and/or acute exacerbations of COPD..$^{57,59,60}$ Other outcomes, such as health status or health-related quality of life and dyspnea, were included in most of these studies. In a study directly comparing FP/SAL with TIO, there was no difference in acute exacerbations of COPD or $\mathrm{FEV}_{1}$ between groups. ${ }^{60}$ Further, in other studies that compared FP/SAL + TIO versus TIO alone, or TIO + SAL, ${ }^{57,59}$ there were also no differences in acute exacerbations of COPD; however, one study showed a lower hospitalization rate with triple therapy. ${ }^{57}$ These studies suggest that the benefits of ICS on the frequency of acute exacerbations of COPD may be less in patients who are on optimal bronchodilation, such as a LABA + long-acting muscarinic antagonist combination. Regarding lung function measures, two studies showed TIO + $\mathrm{SAL}$ or $\mathrm{TIO}+\mathrm{FP} / \mathrm{SAL}$ to be superior to TIO alone, and triple therapy provided the greatest improvement in $\mathrm{FEV}_{1}{ }^{56,57}$ 


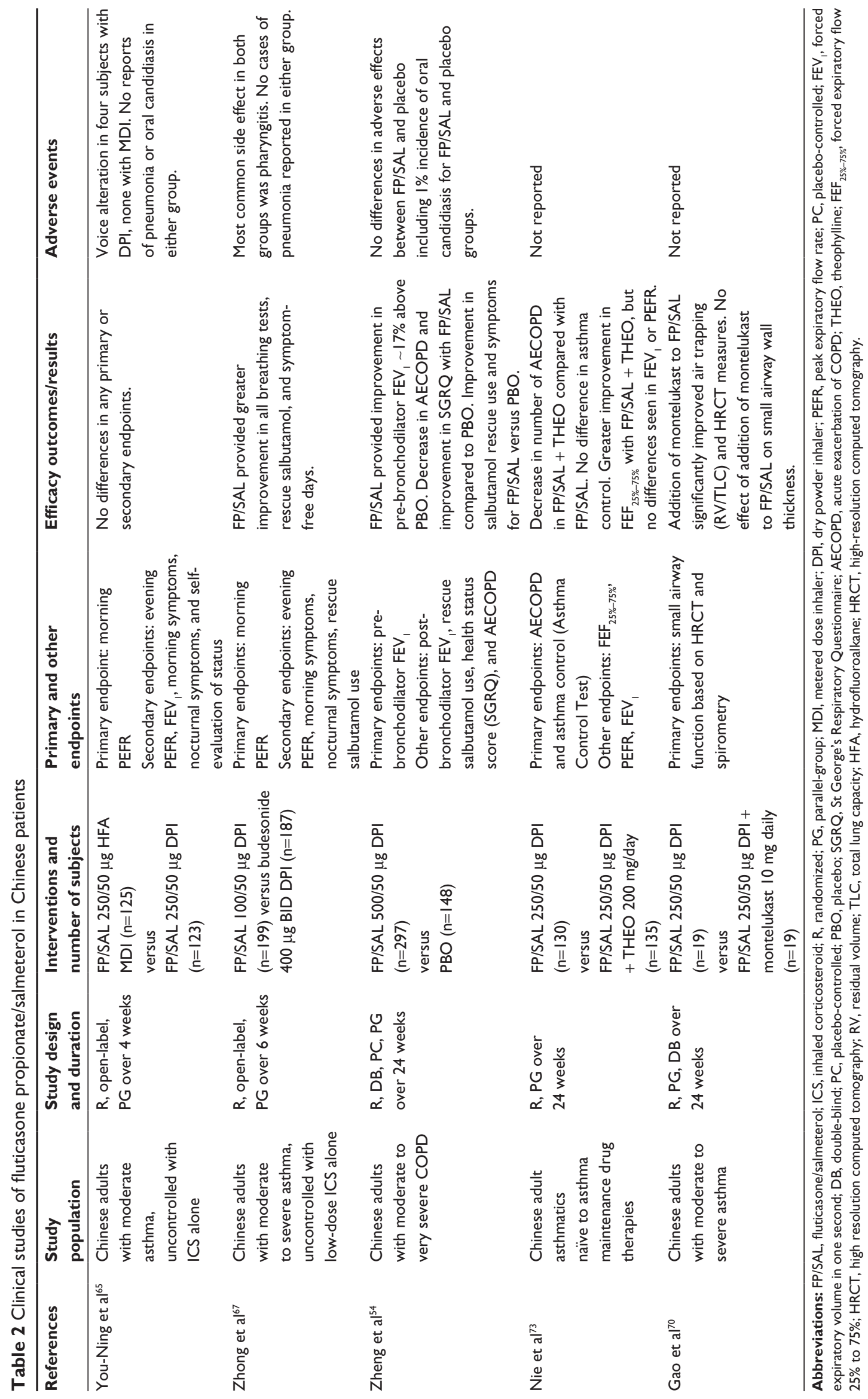




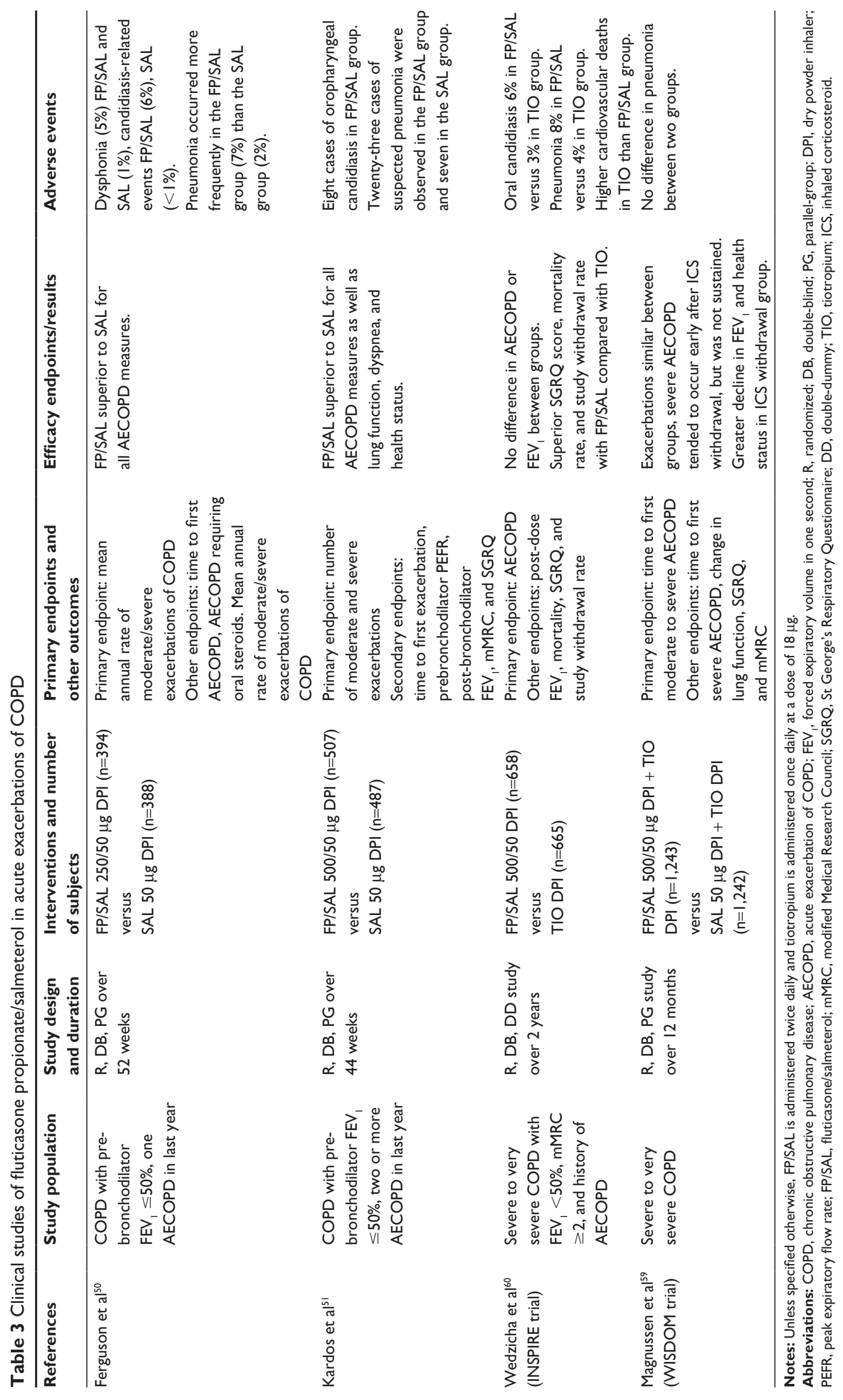




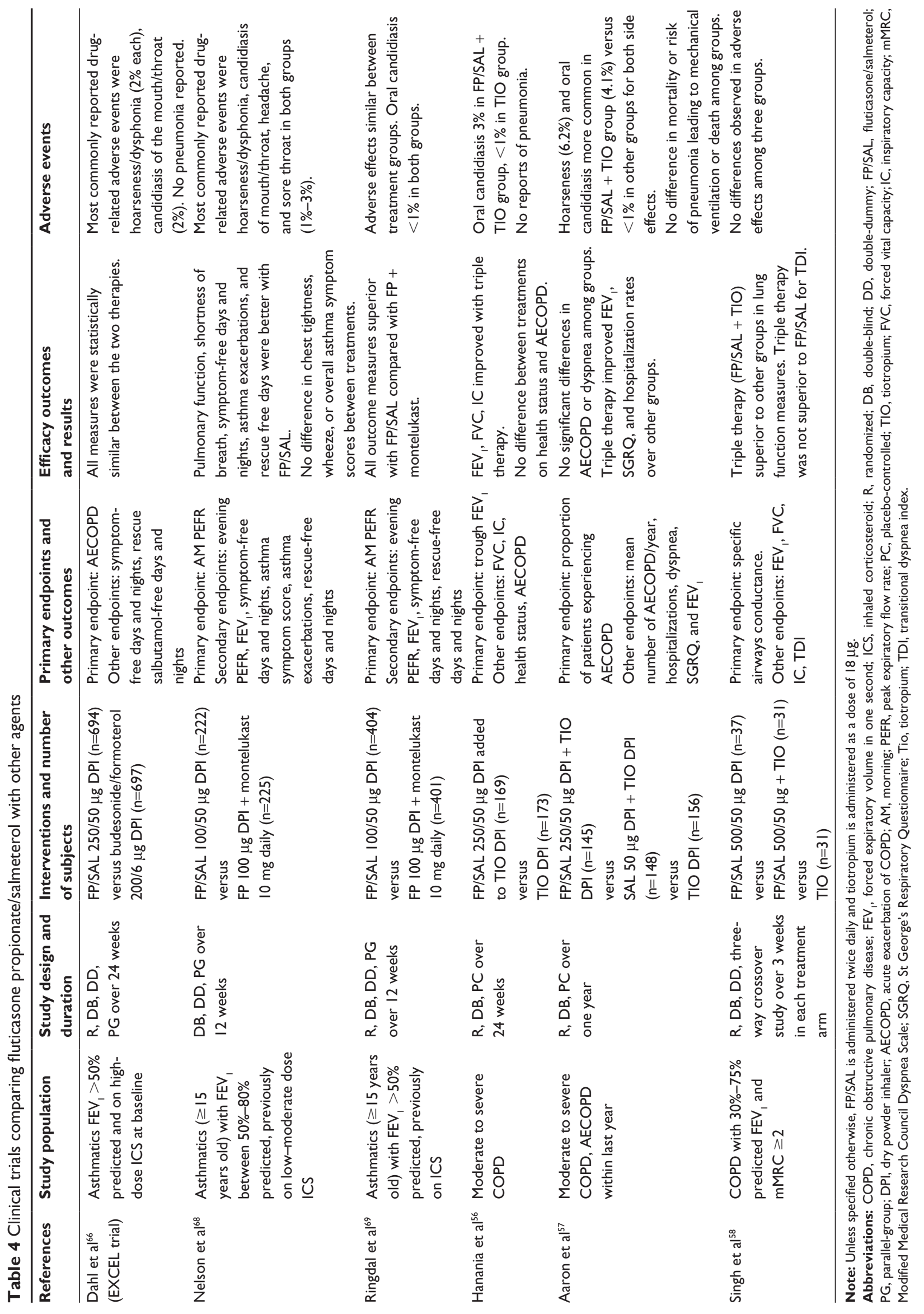


In a recent study, FP/SAL + TIO was superior to SAL + TIO in terms of improvement in lung function. ${ }^{59}$ There were inconsistent findings among the studies of TIO + $\mathrm{FP} / \mathrm{SAL}$ with regard to health-related quality of life $\mathrm{e}^{56,57}$ or dyspnea $^{57-59}$ from TIO alone or TIO + SA.

\section{Clinical trials of FP/SAL in asthma}

In asthma patients with uncontrolled symptoms, twice-daily FP/SAL maintenance therapy improves asthma symptoms and lung function to a greater extent than monotherapy with ICS, such as FP, montelukast with FP, or sustained-release theophylline plus FP. ${ }^{2}$ Two pivotal trials compared FP/ SAL with its individual ingredients and with placebo over 12 weeks in patients with moderate to severe asthma (Table 1). ${ }^{61,62}$ In these studies, FP/SAL was superior to the individual agents as well as placebo. In another pivotal clinical trial in asthmatics requiring high doses of ICS, similar results were found, showing FP/SAL combination to be equivalent to the individual agents. ${ }^{63}$ It was also shown that the efficacy achieved with FP/SAL versus FP alone was sustained for one year in the GOAL (Gaining Optimal Asthma ControL) study of suboptimally controlled asthmatics. ${ }^{14}$ More asthma patients across all severity levels achieved total or better control in then FP/SAL group than those in FP group. Control of asthma symptoms was achieved more rapidly at a lower corticosteroid dose with FP/SAL than with FP alone. Exacerbation rates and improvement in health status were significantly better with FP/SAL. Post hoc analysis of the GOAL study showed that most patients achieving guideline-based control were able to maintain better control with regular, stable dosing, with a low probability of losing control of asthma symptoms. ${ }^{15}$

A recent study of 806 patients compared the effect of a novel once-daily combination of fluticasone furoate, an ICS, and vilanterol, a LABA (FF/VI 100/25 $\mu \mathrm{g}$ once daily) with that of FP/SAL $(250 / 50 \mu \mathrm{g}$, twice daily) in patients with chronic asthma uncontrolled on a medium dose of ICS. ${ }^{64}$ After 24 weeks of treatment, the efficacy of FF/VI was similar to that of FP/SAL in improving lung function over 24 hours (341 $\mathrm{mL}$ increase in $\mathrm{FEV}_{1}$ from baseline in the FF/VI group). There were no differences in asthma control, quality of life questionnaire scores, or reported exacerbations between the FF/VI and FP/SAL treatment groups.

A study performed in Chinese patients with moderate severity asthma uncontrolled with ICS compared the efficacy and safety of FP 125/SAL $25 \mu \mathrm{g}$ (two puffs, twice daily) delivered via an MDI and FP/SAL 250/50 $\mu \mathrm{g}$ delivered via the Diskus DPI (Table 2). ${ }^{65}$ The study endpoints included morning and evening peak expiratory flow rate, $\mathrm{FEV}_{1}$, day-time and nighttime symptom scores, rescue inhaler use, and patient assessment of efficacy. The results showed that FP/SAL delivered by either MDI or Diskus inhaler significantly improved morning peak expiratory flow from baseline.

\section{Comparison of FP/SAL with other ICS/LABAs}

In EXCEL (A randomised trial comparing salmeterol/ fluticasone propionate and formoterol/budesonide combinations in adults with persistent asthma), Dahl et al compared the FP/SAL 250/50 $\mu \mathrm{g}$ DPI with the budesonide/formoterol 200/6 $\mu$ g DPI $\left(\right.$ Symbicort $^{\circledR}$ ) in a group of asthmatics on high-dose ICS with an $\mathrm{FEV}_{1}>50 \%$ (Table 2) ${ }^{66}$ During this 24-week study, there was no difference in acute exacerbations of COPD, symptom-free days and nights, or rescue inhaler-free days and nights. This suggests that these two ICS/LABA combinations, both of which are available in the People's Republic of China, are equally efficacious in adult asthmatics.

In 2004, Zhong et al compared the efficacy and safety of low-dose FP/SAL (100/50 $\mu \mathrm{g}$, twice daily) combination therapy with that of a moderate dose of budesonide $(400 \mu \mathrm{g}$, twice daily) in the management of Chinese adults with asthma (Table 2) ${ }^{67}$ Over the 6-week treatment period, FP/SAL significantly reduced the need for rescue medication, increased morning and evening peak expiratory flow measurements, and improved day-time and nighttime asthma symptoms when compared with budesonide alone. Low-dose FP/SAL was a better choice for the control of asthma in Chinese patients, and addition of a LABA to ICS was superior to an increased dosage of ICS.

\section{Comparison of FP/SAL with montelukast in asthma}

There are several published studies comparing montelukastICS combinations with FP/SAL in adults with asthma (Table 4), ${ }^{68,69}$ including one study in Chinese patients ${ }^{70}$ (Table 3). Two clinical trials compared the effects of FP/SAL and FP + montelukast $10 \mathrm{mg}$ daily over 12 weeks on lung function measures and asthma control in patients aged 15 years or older. ${ }^{68,69}$ Both trials found that FP/SAL was superior to $\mathrm{FP}+$ montelukast with regard to lung function measures, overall asthma control, and exacerbations. ${ }^{68,69}$ The primary outcome in the Chinese study was to compare the effect of montelukast + FP/SAL versus FP/SAL on small airway function. ${ }^{70}$ Using computed tomography, patients receiving $\mathrm{FP} / \mathrm{SAL}+$ montelukast showed significant improvement in the ratio of computed tomography-determined values at full 
expiration to those at full inspiration when compared with those receiving $\mathrm{FP} / \mathrm{SAL}$.

\section{Comparison of FP/SAL with theophylline in asthma}

Theophylline, a member of the methylxanthines, has been used as a treatment for both asthma and COPD for many years, ${ }^{71}$ particularly in the People's Republic of China. GINA and GOLD guidelines consider theophylline as an add-on agent to therapies such as ICS/LABA. Today, target theophylline blood levels are lower than previously utilized $(5-10 \mu \mathrm{g} / \mathrm{mL}$ versus $10-20 \mu \mathrm{g} / \mathrm{mL}$, respectively), and so theophylline is now better tolerated and less subject to significant drug interactions. ${ }^{72}$ In addition to its bronchodilatory and anti-inflammatory effects, theophylline may help to reverse the steroid resistance related to histone deacetylase activity in smokers. ${ }^{71}$

There are two published clinical studies of theophylline in Chinese asthmatics receiving an ICS with or without a LABA; however, there are no such published studies for COPD in the People's Republic of China (Table 2). ${ }^{73,74}$ One study showed that theophylline provides benefit in asthmatics using FP/SAL. In a study of 325 Chinese asthmatics, there were significantly fewer patients with one or more exacerbations in the theophylline + FP/SAL group compared with the FP/ $\mathrm{SAL}+$ placebo group $(29.6 \%$ versus $46.9 \%, P=0.004)$. Theophylline $+\mathrm{FP} / \mathrm{SAL}$ improved small airway dysfunction to a greater extent than FP/SAL + placebo (66.9\% versus $57.4 \%$, $P<0.001)$. A significant decrease in eosinophil count and concentration of sputum eosinophilic cationic protein was also seen in the theophylline $+\mathrm{FP} / \mathrm{SAL}$ combination group. ${ }^{73}$

In another study of 41 Chinese patients with asthma, Wang et al compared the efficacy of ICS combined with sustained-release theophylline versus doubling the dose of ICS on asthma control and anti-inflammatory activity. ${ }^{74}$ Compared with double-dose ICS, low-dose ICS + theophylline significantly increased the morning and evening peak expiratory flow rate and $\mathrm{FEV}_{1}$, and reduced asthma symptom scores and use of inhaled $\beta_{2}$-agonists. Low-dose ICS in combination with theophylline significantly decreased eosinophil and interleukin-5 levels in sputum.

\section{Tolerability}

In general, FP is well tolerated in adults, with the most frequent drug-related adverse events being related to the topical effects of ICS, including dysphonia, oral/esophageal candidiasis, and throat irritation. Local side effects from ICS are a function of steroid potency and the amount of drug deposited in the oropharyngeal cavity, the latter largely a function of inhalant particle size, device, patient technique, and oral hygiene. Clinical studies in Table 1 show that the prevalence of oral candidiasis ranges from $<3 \%$ to $10 \%{ }^{9,13,14,67}$ and dysphonia from $0 \%$ to $17 \%{ }^{11,61}$ The FP/SAL DPI has a relatively large particle size compared with some of the other inhalers, and this may result in lower lung and higher oropharyngeal deposition rates. You-Ning et al found a higher rate of dysphonia with the FP/SAL DPI than with the FP/SAL hydrofluoroalkane MDI in a group of Chinese asthmatics. ${ }^{65}$ One disadvantage of the FP/SAL DPI is the inability to use a spacer to decrease oropharyngeal deposition; alternatively, the FP/SAL hydrofluoroalkane (HFA) MDI or another ICS/LABA MDI could be used in conjunction with a spacer to decrease oropharyngeal deposition and therefore local side effects.

An increased risk of pneumonia has been reported in COPD patients treated with FP,,$^{11,50,60,75,76}$ but not in other studies..$^{55,57,59}$ However, the risk appears to be very low in asthmatics, ${ }^{77}$ although this requires additional study, especially in older asthmatics. It is unclear whether this is a local and/or systemic effect with ICS. Although reported in earlier studies, the high rates of pneumonia with FP-containing regimens in the TORCH trial $(>18 \%)$ highlighted this potential adverse effect, ${ }^{11}$ and a number of subsequent studies have also reported on this adverse effect. However, in the TORCH study, the higher risk of pneumonia with FP did not appear to affect overall mortality, with 439 deaths occurring in subjects receiving FP compared with 436 deaths in subjects not receiving FP. ${ }^{11} \mathrm{~A}$ post hoc analysis of the TORCH trial reported that COPD patients with a low body mass index, $\mathrm{FEV}_{1}<50 \%$, age $>$ 55 years, and recent exacerbations had an increased the risk of pneumonia associated with FP. ${ }^{75}$ Some studies report a higher risk of pneumonia in COPD patients receiving FP/SAL than in those receiving tiotropium, ${ }^{56,60}$ whereas other studies show no increased risk. ${ }^{57,59}$ A retrospective Canadian study by Suissa et al suggested that the risk of pneumonia with FP is dose-related..$^{76}$ Additional research is needed to better understand the relationship between ICS and the risk of pneumonia, primarily in COPD, but also in asthma.

\section{Inhaler delivery devices for FP/SAL}

Currently, FP/SAL is available in the Diskus ${ }^{\circledR}$ DPI, also known as the Accuhaler ${ }^{\circledR}$ in some parts of the world, including in the People's Republic of China, and in a HFA MDI worldwide. The Diskus ${ }^{\circledR}$ was the first device available for FP/ SAL, then later the MDI. The Diskus/Accuhaler ${ }^{\circledR}$ accounts for the majority of use of FP/SAL inhalers in patients with asthma and COPD. In general, DPIs are easier for patients to use 
than MDIs, as the former are breath-activated. A recent study evaluating patients' ability to use different inhalers showed that the Diskus ${ }^{\circledR}$ was one of the easiest devices for patients to use. ${ }^{78}$ A study specifically done in the Chinese population with the Accuhaler showed that the most common error made by patients was that patients cannot take a full deep inhalation. The authors pointed to the need for patient education on proper inhalation technique with this device. ${ }^{79}$ Another study determined the effect of advanced COPD on achieving adequate inspiratory effort using the Diskus ${ }^{\circledR} .{ }^{80}$ They found that $20 \%$ of COPD patients were unable to achieve an inspiratory rate of $>60 / \mathrm{min}$, particularly in females of short stature with low FVC and poor peak inspiratory flow rates.

\section{Summary}

COPD and asthma are widely prevalent diseases in the People's Republic of China, with environmental risk factors interacting with genetic susceptibility to influence the expression and progression of these diseases. Importantly, the direct and indirect costs of these two diseases are high in both rural and urban areas of the People's Republic of China, particularly in the former. Their diagnosis and management are too frequently not in accordance with the GOLD and GINA recommendations, likely related to providers' inadequate awareness of the details of published guidelines, economic considerations, lack of availability of spirometry, suboptimal prescribing behaviors, and the challenges associated with busy clinical practice settings.

Inhaled FP/SAL has become a mainstay for the longterm management of asthma and COPD. Published studies have shown this combination product to be effective in the Chinese population for the treatment of asthma and COPD. These studies show efficacy similar to that in investigations conducted in Western patient populations, including improvements in $\mathrm{FEV}_{1}$, a decrease in acute exacerbations of COPD, and improvement in quality of life. Adverse effects such as oral candidiasis and pneumonia were infrequently reported in the Chinese studies. The Accuhaler ${ }^{\circledR}$ (Diskus) DPI is simple and convenient for patients to use, although proper technique should be ensured when initiating therapy. Additional studies of ICS/LABA combination therapy in Chinese patients are needed to determine their efficacy in COPD/asthma overlap syndrome, clarify the potential benefits with regard to mortality, and better define the risks of pneumonia and long-term adverse effects.

\section{Acknowledgment}

This work was partly supported by grants from the Natural Sciences Foundation of China (81170040, 81470229),
National Science and Technology Pillar Program during the Twelfth Five-Year Period (2012BAI05B00), and the Peking Union Medical College.

\section{Disclosure}

Dr Pleasants conducts Clinical Research for GSK. The authors report no conflicts of interest in this work.

\section{References}

1. Global Initiative for Chronic Obstructive Lung Disease. Global strategy for the diagnosis, management, and prevention of chronic obstructive pulmonary disease. Available from: http://www.goldcopd.org/uploads/ users/files/GOLD_Report_2014_Jun11.pdf. Accessed June 18, 2014.

2. Global Initiative for Asthma. Global strategy for asthma management and prevention. Available from: http://www.ginasthma.org/local/uploads/ files/GINA_Report_2014_Jun11.pdf. Accessed June 18, 2014.

3. Page C, Cazzola M. Bifunctional drugs for the treatment of asthma and chronic obstructive pulmonary disease. Eur Respir J. 2014;44:475-482.

4. Frois C, Wu EQ, Ray S, Colice GL. Inhaled corticosteroids or long-acting beta-agonists alone or in fixed-dose combinations in asthma treatment: a systematic review of fluticasone/budesonide and formoterol/salmeterol. Clin Ther. 2009;31:2779-2803.

5. Tamm M, Richards DH, Beghé B, Fabbri L. Inhaled corticosteroid and long-acting $\beta 2$-agonist pharmacological profiles: effective asthma therapy in practice. Respir Med. 2012;106 Suppl 1:S9-S19.

6. McKeage K, Keam SJ. Salmeterol/fluticasone propionate: a review of its use in asthma. Drugs. 2009;69:1799-1828.

7. Chung KF. Salmeterol/fluticasone combination in the treatment of COPD. Int J COPD. 2006;1:235-242.

8. Tashkin DP, Ferguson GT. Combination bronchodilator therapy in the management of chronic obstructive pulmonary disease. Respir Res. 2013; $14: 49$.

9. Mahler DA, Wire P, Horstman D, et al. Effectiveness of fluticasone propionate and salmeterol combination delivered via the Diskus device in the treatment of chronic obstructive pulmonary disease. Am J Respir Crit Care Med. 2002;166:1084-1091.

10. Calverley P, Pauwels R, Vestbo J, et al; TRial of Inhaled STeroids ANd long-acting beta2 agonists study group. Combined salmeterol and fluticasone in the treatment of chronic obstructive pulmonary disease: a randomised controlled trial. Lancet. 2003;361:449-456.

11. Calverley PMA, Anderson JA, Celli B, et al; for the TORCH investigators. Salmeterol and fluticasone propionate and survival in chronic obstructive pulmonary disease. $N$ Engl J Med. 2007;356:755-789.

12. Celli BR, Thomas NE, Anderson JA, et al. Effect of pharmacotherapy on rate of decline of lung function in chronic obstructive pulmonary disease: results from the TORCH study. Am J Respir Crit Care Med. 2008; 178:332-338.

13. Hanania NA, Darken P, Horstman D, et al. The efficacy and safety of fluticasone propionate (250 microg)/salmeterol (50 microg) combined in the Diskus inhaler for the treatment of COPD. Chest. 2003;124: 834-843.

14. Bateman ED, Boushey HA, Bousquet J, et al; for the GOAL Investigators Group. Can guideline-defined asthma control be achieved? The Gaining Optimal Asthma controL study. Am J Respir Crit Care Med. 2004;170: 836-844.

15. Bateman ED, Bousquet J, Busse WW, et al; on behalf of the GOAL Steering Committee and Investigators. Stability of asthma control with regular treatment: an analysis of the Gaining Optimal Asthma controL (GOAL) study. Allergy. 2008;63:932-938.

16. Global Initiative for Chronic Obstructive Lung Disease. Diagnosis of diseases of chronic airflow limitation: asthma, COPD and asthma-COPD overlap syndrome (ACOS), May 2014. Available from: http://www. goldcopd.org/uploads/users/files/AsthmaCOPDOverlap.pdf. Accessed March 7, 2015. 
17. Lopez AD, Murray CC. The global burden of disease, 1990-2020. Nat Med. 1998;4(11):1241-1243.

18. Respiratory Diseases Branch of Chinese Medical Association. A national guideline for the diagnosis, management, and prevention of chronic obstructive pulmonary disease in China. Chin J Tuberc Respir Dis. 2007;30:8-17.

19. Zhong N, Wang C, Yao W, et al. Prevalence of chronic obstructive pulmonary disease in China: a large, population-based survey. $\mathrm{Am}$ J Respir Crit Care Med. 2007;176:753-760.

20. Chinese Ministry of Health. The top ten disease specific rates and causes of death among urban and rural residents. Available from: http://www. moh.gov.cn/publicfiles/business/htmlfiles/zwgkat/ptjnj/20008/42835. htm. Accessed June 1, 2014.

21. Fang X, Wang X, Bai C. COPD in China: the burden and importance of proper management. Chest. 2011;139:920-929.

22. Lin HH, Murray M, Cohen T, Colijn C, Ezzati M. Effects of smoking and solid-fuel use on COPD, lung cancer, and tuberculosis in China: a time-based, multiple risk factor, modelling study. Lancet. 2008;372: 1473-1483.

23. Lu P, Zhu Y, Chen P, et al. Vulnerability of patients with chronic obstructive pulmonary disease according to gender in China. Int $J$ COPD. 2012;7:825-832.

24. Li Q, Hsia J, Yang G. Prevalence of smoking in China in 2010. N Engl J Med. 2011;364:2469-2470.

25. Yin P, Zhang M, Li Y, Jang Y, Zhao W. Prevalence of COPD and its association with socioeconomic status in China: findings from China Chronic Disease Risk Factor Surveillance. BMC Public Health. 2011; 11:586.

26. To T, Stanojevic S, Moores G, et al. Global asthma prevalence in adults: findings from the cross-sectional world health survey. BMC Public Health. 2012;12:204.

27. Leung R, Wong G, Lau J, et al. Prevalence of asthma and allergy in Hong Kong schoolchildren: an ISAAC study. Eur Respir J. 1997;10: 354-360.

28. Wong GW, Ko FW, Hui DS, et al. Factors associated with difference in prevalence of asthma in children from three cities in China: multicentre epidemiological survey. BMJ. 2004;329:486.

29. International Study of Asthma and Allergies in Childhood Steering Committee. Worldwide variation in prevalence of symptoms of asthma, allergic rhinoconjunctivitis, and atopic eczema. Lancet. 1998; 351:1125-1132.

30. Brooks C, Pearce N, Douwes J. The hygiene hypothesis in allergy and asthma: an update. Curr Opin Allergy Clin Immunol. 2013;13:70-77.

31. He QY, Zhou X, Xie CM, et al. [Impact of chronic obstructive pulmonary disease on quality of life and economic burden in Chinese urban areas]. Zhonghua Jie He He Hu Xi Za Zhi. 2009;32:253-257. Chinese.

32. Lou P, Zhu Y, Chen P, et al. Vulnerability, beliefs, treatments and economic burden of chronic obstructive pulmonary disease in rural areas in China: a cross-sectional study. BMC Public Health. 2012;12:287.

33. Respiratory Diseases Branch of the Chinese Medical Association. [Bronchial asthma therapeutic guidelines. Definition, diagnosis, treatment, education and management of bronchial asthma]. Chin J Tuberc Respir Dis. 2003;26:132-138. Chinese.

34. Li ZP, Huang JQ, Tang KJ. [Retrospective studies on 713 cases chronic obstructive pulmonary disease]. Zhonghua Liu Xing Bing Xue Za Zhi. 2003;24:722-724. Chinese.

35. Shen N, Yao WZ, Zhu H. [Patient's perspective of chronic obstructive pulmonary disease in Yanqing county of Beijing]. Zhonghua Jie He He Hu Xi Za Zhi. 2008;31:206-208. Chinese.

36. Zhang RB, He QY. [Awareness of knowledge of COPD by doctors in district and community hospitals]. Chin J Prev Control Chronic Dis. 2009;17:61-63. Chinese.

37. He QY, Zhou X, Xie CM, et al. [The investigation of the treatment conditions in stable COPD patients in partial cities in People's Republic of China]. Chin J Pract Intern Med. 2009;29:354-357. Chinese.

38. Su N, Lin J, Chen P, et al. Evaluation of asthma control: a questionnairebased survey in China. Chin Med J (Engl). 2014;127:2030-2036.
39. Barnes NC, Qiu YS, Pavord I, et al. Anti-inflammatory effects of salmeterol/fluticasone propionate in chronic obstructive lung disease. Am J Respir Crit Care Med. 2006;173:736-743.

40. Sin DD, Lacy P, York E, et al. Effects of fluticasone on systemic markers of inflammation in chronic obstructive pulmonary disease. Am J Respir Crit Care Med. 2004;170:760-765.

41. Sin DD, Man SFP, Marciniuk DD, et al; for the ABC (Advair, Biomarkers in COPD) Investigators. The effects of fluticasone with or without salmeterol on systemic biomarkers of inflammation in chronic obstructive pulmonary disease. Am J Respir Crit Care Med. 2008;177:1207-1214.

42. Yang L, Ma Q, Yao W, et al. Relationship between the anti-inflammatory properties of salmeterol/fluticasone and the expression of CD4+CD25+Foxp3+ regulatory T cells in COPD. Respir Res. 2011;12: 142.

43. Tang YJ, Wang K, Yuan T, et al. Salmeterol/fluticasone treatment reduces circulating C-reactive protein level in patients with stable chronic obstructive pulmonary disease. Chin Med J (Engl). 2010;123:1652-1657.

44. Du Y, Wang W, Yang W, et al. Interleukin-32, not reduced by salmeterol/fluticasone propionate in smokers with chronic obstructive pulmonary disease. Chin Med J (Engl). 2014;127:1613-1618.

45. Currie GP, Syme-Grant NJ, McFarlane LC, Carey FA, Lipworth BJ. Effects of low dose fluticasone/salmeterol combination on surrogate inflammatory markers in moderate persistent asthma. Allergy. 2003; 58:602-607.

46. Pavord I, Woodcock A, Parker D, et al; for the SOLTA Study Group. Salmeterol plus fluticasone propionate versus fluticasone propionate plus montelukast: a randomised controlled trial investigating the effects on airway inflammation in asthma. Respir Res. 2007;8:67.

47. Chen P, Zhao HT, Sun L, et al. [The efficacy of half of the Global Initiative for Asthma recommended dose of inhaled corticosteroids in the management of Chinese asthmatics]. Chinese. Zhonghua Jie He He Hu Xi Za Zhi. 2005;28(7):458-463. Chinese.

48. Boyd G, Morice AH, Pounsford JC, et al. An evaluation of salmeterol in the treatment of chronic obstructive pulmonary disease (COPD). Eur Respir J. 1997;10:815-821.

49. Ulrik CS. Efficacy of inhaled salmeterol in the management of smokers with chronic obstructive pulmonary disease: a single centre randomised, double blind, placebo controlled, crossover study. Thorax. 1995;50:750-754.

50. Ferguson G, Anzueto A, Fei R, et al. Effect of fluticasone propionate/ salmeterol $(250 / 50 \mathrm{mcg})$ or salmeterol $(50 \mathrm{mcg})$ on COPD exacerbations. Respir Med. 2008;102:1099-1108.

51. Kardos P, Wencker M, Glaab T, Vogelmeier C. Impact of salmeterol/ fluticasone propionate versus salmeterol on exacerbations in severe chronic obstructive pulmonary disease. Am J Respir Crit Care Med.2007; 175:144-149.

52. Vestbo J, Anderson JA, Calverley PM, et al. Adherence to inhaled therapy, and mortality and hospital admission for COPD. Thorax. 2009; 64:939-943.

53. Jenkins CR, Jones P, Calverley PM, et al. Efficacy of salmeterol/ fluticasone propionate by GOLD stage of chronic obstructive pulmonary disease: analysis from the randomised, placebo-controlled TORCH study. Respir Res. 2009;10:59.

54. Zheng JP, Yang L, Wu YM, et al. The efficacy and safety of combination salmeterol $(50 \mu \mathrm{g}) /$ fluticasone propionate $(500 \mu \mathrm{g})$ inhalation twice daily via accuhaler in Chinese patients with COPD. Chest. 2007;132: 1756-1763.

55. Cheng S, Su K, Wang K, Peng D, Yang P. Chronic obstructive pulmonary disease treated with inhaled medium- or high-dose corticosteroids: a prospective and randomized study focusing on clinical efficacy and the risk of pneumonia. Drug Des Devel Ther. 2014;8:601-607.

56. Hanania N, Crater G, Morris A, et al. Benefits of adding fluticasone propionate/salmeterol to tiotropium in moderate to severe COPD. Respir Med. 2012;106:91-101.

57. Aaron SD, Vandemheen K, Ferguson D, et al. Tiotropium in combination with placebo, salmeterol, or fluticasone/salmeterol for treatment of COPD: a randomized trial. Ann Intern Med. 2007;146:545-555. 
58. Singh D, Brooks J, Hagan G, Cahn A, Connor J. Superiority of "triple" therapy with salmeterol/fluticasone propionate and tiotropium bromide versus individual components in moderate to severe COPD. Thorax. 2008;63:592-598.

59. Magnussen H, Disse B, Rodriguez-Roisin R, et al. Withdrawal of inhaled glucocorticoids and exacerbations of COPD. $N$ Engl J Med. 2014;371:1285-1294.

60. Wedzicha JA, Calverley PM, Seemungal TA, et al. The prevention of chronic obstructive pulmonary disease exacerbations by salmeterol/fluticasone propionate or tiotropium bromide. Am J Respir Crit Care Med. 2008;177:19-26

61. Shapiro G, Shapiro G, Lumry W, et al. A Combined salmeterol $50 \mathrm{mg}$ and fluticasone propionate $250 \mathrm{mg}$ in the Diskus device for the treatment of asthma. Am J Respir Crit Care Med. 2000;161:527-534.

62. Kavuru M, Melamed J, Gross G, et al. Salmeterol and fluticasone propionate combined in a new powder inhalation device for the treatment of asthma: a randomized, double-blind, placebo-controlled trial. J Allergy Clin Immunol. 2000;105:1108-1116.

63. Aubier M, Pieters W, Schlosser N, et al. Salmeterol/fluticasone propionate $(50 / 500 \mu \mathrm{g})$ in combination in a Diskus ${ }^{\circledR}$ inhaler $\left(\right.$ Seretide $^{\circledR}$ ) is effective and safe in the treatment of steroid-dependent asthma. Respir Med. 1999;93:876-884.

64. Woodcock A, Bleecker ER, Lötvall J, et al. Efficacy and safety of fluticasone furoate/vilanterol compared with fluticasone propionate/ salmeterol combination in adult and adolescent patients with persistent asthma: a randomized trial. Chest. 2013;144:1222-1229.

65. You-Ning L, Humphries M, Du X, Wang L, Jiang J. Efficacy and safety of salmeterol/fluticasone propionate delivered via a hydrofluoroalkane metered dose inhaler in Chinese patients with moderate asthma poorly controlled with inhaled corticosteroids. Int J Clin Pract. 2005;59: 754-759.

66. Dahl R, Chuchalin A, Gor D, et al. EXCEL: a randomized trial comparing salmeterol/fluticasone propionate and formoterol/budesonide combinations in adults with persistent asthma. Respir Med. 2006;100: 1152-1162.

67. Zhong NS, Zheng JP, Cai BQ, et al. Salmeterol/fluticasone propionate in a single inhaler is superior to budesonide alone in control of Chinese asthmatic adults. Clin Drug Investig. 2004;24:583-594.
68. Nelson H, Busse W, Kerwin E, et al. Fluticasone propionate/salmeterol combination provides more effective asthma control than low-dose inhaled corticosteroid plus montelukast. J Allergy Clin Immunol. 2002; 106:1088-1095.

69. Ringdal N, Eliraz A, Pruzinec P, et al. The salmeterol/fluticasone combination is more effective than fluticasone plus oral montelukast in asthma. Respir Med. 2003;97:234-241.

70. Gao J, Feng C, Peng M, Ma Y, Wang B. Montelukast improves air trapping, not airway remodeling, in patients with moderate-to-severe asthma: a pilot study. Chin Med J (Engl). 2013;126:2229-2234.

71. Barnes PJ. Theophylline: new perspectives for an old drug. Am J Respir Crit Care Med. 2003;167:813-818.

72. Barnes P. Theophylline. Pharmaceuticals (Basel). 2010;3:725-747.

73. Nie H, Zhang G, Liu M, et al. Efficacy of theophylline plus salmeterol/ fluticasone propionate combination therapy in patients with asthma. Respir Med. 2013;107:347-354.

74. Wang Y, Wang CZ, Lin KX, et al. Comparison of inhaled corticosteroid combined with theophylline and double-dose inhaled corticosteroid in moderate to severe asthma. Respirology. 2005;10:189-195.

75. Crim C, Calverley PMA, Anderson JA, et al. Pneumonia risk in COPD patients receiving inhaled corticosteroids alone or in combination: TORCH study results. Eur Respir J. 2009;34:641-647.

76. Suissa S, Patenaude V, Lapi F, Ernest P. Inhaled corticosteroids in COPD and the risk of serious pneumonia. Thorax. 2013;68:1029-1036.

77. O'Byrne P, Pedersen S, Carlsson L, et al. Risks of pneumonia in patients with asthma taking inhaled corticosteroids. Am J Respir Crit Care Med. 2011;183:589-595.

78. Chorão P, Pereira AM, Fonseca JA. Inhaler devices in asthma and COPD - an assessment of inhaler technique and patient preferences. Respir Med. 2014;108:968-975.

79. Li H, Chen Y, Zhang Z, Dong X, Zhang G, Zhang H. Handling of Diskus dry powder inhaler in Chinese chronic obstructive pulmonary disease patients. Aerosol Med Pulm Drug Deliv. 2014;27:219-227.

80. Mahler DA, Waterman LA, Gifford AH. Prevalence and COPD phenotype for a suboptimal peak inspiratory flow rate against the simulated resistance of the Diskus ${ }^{\circledR}$ dry powder inhaler. J Aerosol Med Pulm Drug Deliv. 2013;26:174-179.
International Journal of COPD

\section{Publish your work in this journal}

The International Journal of COPD is an international, peer-reviewed journal of therapeutics and pharmacology focusing on concise rapid reporting of clinical studies and reviews in COPD. Special focus is given to the pathophysiological processes underlying the disease, intervention programs, patient focused education, and self management protocols.

\section{Dovepress}

This journal is indexed on PubMed Central, MedLine and CAS. The manuscript management system is completely online and includes a very quick and fair peer-review system, which is all easy to use. Visit http://www.dovepress.com/testimonials.php to read real quotes from published authors. 